\title{
Controllability of Fractional-order Dynamical System with Time Varying-Delays
}

\author{
${ }^{1}$ Jackreece P. C., ${ }^{2}$ Godspower, C. A., ${ }^{3}$ Ugorji, H. C. \\ ${ }^{1,2,3}$ Department of Mathematics and Statistics- University of Port Harcourt- Port Harcourt- Nigeria \\ prebo. jackreece@uniport. edu.ng, ${ }^{2}$ fabulousgp@ymail. com, ${ }^{3}$ hycinth. ugorji@uniport. edu.ng
}

\begin{abstract}
In this study, controllability of a class of fractional-order dynamical system with time-varying delays is considered. First, a representation of the solution is given using Laplace transform and Mittageleffler function. Then necessary and sufficient conditions for the controllability of the fractional order system are derived by using algebraic criterion.
\end{abstract}

Keywords: Fractional order system, time varying delays, Laplace transform, Mittage-Leffler function, controllability, algebraic criteria

2010 MSC: 93B05, 34A08, 33E12, 44A10

\section{Introduction}

Currently, qualitative properties such as observability, controllability, stability, stabilizability of fractional-order dynamical systems are the issues dealt with by scholars. A fundamental concept widely used in the analysis and design of control systems in modern control theory is Controllability. (see [9], [30]). Controllability is the possibility of steering a dynamical system from an arbitrary initial state to an arbitrary final (desired) state by using a set of admissible controls. Depending on the type of dynamical system, there are numerous types of definitions of controllability [9], [24], [30].

Numerous remarkable results concerning the controllability of different types of dynamical systems have been obtained in ([14], [23], [26], [24], [25], [28], [19] and references therein). Different techniques have been used in establishing controllability of linear and nonlinear dynamical systems. [44], [47], [28], [27], [22], [29], [3], used fixed-point techniques to derive sufficient conditions for the controllability of dynamical systems. [12], [1], [4], [5] used the measure of noncompactness of a set and fixed-point theorem due to Darbo in establishing controllability results of nonlinear systems with implicit derivatives. [20], [17] used algebraic methods. [43] used geometric approach, while [11], [32], [16] used functional analytic method.

The development of fractional-order calculus since the $17^{\text {th }}$ century can be traced to the works of Leibnitz, Liouville, Riemann, Grunwald, and Letnikov [21], [36], [40]. Fractional-order calculus is an extension of the classical calculus in which the order of the derivatives and integrals are of arbitrary order. Recently, due to the important role fractional-order systems plays in various scientific fields such as mathematics, physics, engineering, biology, chemistry, etc [21], [42], [45], [39], [36], [31], a renewed interest by scholars has been devoted to its study. The most important aspects which essentially differentiates fractional-order models and integer-order models are the following. First for mechanical and physical processes, at a particular time, the integer-order derivatives indicate a variation of a certain attributes, while the fractional-order derivative involves the entire space. Second, for physical processes, integer order derivatives describe the local properties of a certain position, while fractional order derivatives describe the nonlocal properties, [10], [18]. However, in many real-world phenomena, fractional order models are more realistic and 
suitable to describe its behaviors than integer order models, for details see [33], [46], [35], [38], [18], [15].

The study of controllability of fractional-order dynamical systems provide important issues for many applied problems due to the use of fractional order derivatives and integrals in control theory leading to better results than the integer ones. In recent years, the controllability of fractionalorder dynamical systems has attracted the attention of many researchers because of the critical role it plays in their analysis. [41] established different types of necessary and sufficient conditions for the relative controllability and relative constrained controllability for both with and without retarded state for linear fractional-order systems. In [6] sufficient condition for the controllability of nonlinear integrodifferential system with implicit fractional derivative is obtained using the notion of the measure of noncompactness of a set and Darbo's fixed point theorem, while [2] used Schauder's fixed point theorem to prove sufficient conditions for the controllability of linear and nonlinear fractional-order dynamical systems in finite dimensional spaces. [8] studied sufficient conditions for the relative controllability of nonlinear fractional-order dynamical systems defined in a finite dimensional space with time varying multiple and distributed delays in the control using Schauder,s fixed point theorem. [7] proved controllability of linear and nonlinear fractional damped dynamical system in finite dimensional spaces using Mittage-Leffler matrix function and iterative techniques. Motivated by this literature, this article seeks to prove sufficient conditions for the controllability of a class of fractional-order dynamical systems with time-varying delays in the state defined by

$$
\begin{aligned}
& { }^{C} D_{t}^{\alpha} x(t)=A_{0}(t) x(t)+A_{1}(t) x(t-\sigma(t))+B(t) u(t) \\
& x(0)=x_{0}, \quad \forall t \in\left[-\sigma_{M}, 0\right]
\end{aligned}
$$

where ${ }^{C} D_{t}^{\alpha}$ is the Caputo fractional-order derivative of order $\alpha, \alpha \in(0,1), D$ the classical differential operator, $x(t) \in R^{n}$ is the state vector, $u(t) \in R^{m}$ is the control vector, $A_{0}(t), A_{1}(t)$ and $B(t)$ are matrices of bounded variation of appropriate dimension, $\sigma(t)$ is timevarying delay, $x_{0} \in C\left([-\sigma, 0], R^{n}\right)$ is an admissible initial state and $C\left(\left[-\sigma_{M}, 0\right], R^{n}\right)$ is a Banach space of continuous function $\phi:\left[-\sigma_{M}, 0\right] \rightarrow R^{n}$ which converges uniformly and equipped with standard supremum norm $\|$.$\| given by$

$$
\|\varphi\|=\sup _{-\sigma_{\max } \leq \theta \leq 0}|\varphi(\theta)|
$$

$I=[0, T]$, where $T>0$ is the time interval over which the system's behavior is defined.

\section{Preliminaries}

First we recall some basic definitions, notations, lemmas, theorems and preliminary facts that will be used later in the work, (see [21], [34], [37], [40] for details).

Definition 2.1 Let the function $f: \square^{+} \rightarrow \square$, then the Caputo fractional-order derivative of order $\alpha$ is defined as

$$
{ }^{c} D_{t}^{\alpha} f(t)=\frac{1}{\Gamma(n-\alpha)} \int_{0}^{t} \frac{f^{n}(s)}{(t-s)^{\alpha+1-n}} d s, \alpha \in(n-1, n), \forall n \in \square
$$


where $\Gamma($.$) is the Euler's gamma function, and D_{t}^{\alpha} f(t)=\frac{d^{n} f(t)}{d t^{n}}$ if $\alpha=n$. The nth derivative of order $n$ of the function $f(t)$ with respect to $t$ is denoted by $\frac{d^{n} f(t)}{d t^{n}}$. The Caputo fractional derivative and ordinary derivatives have quite similar properties. Also, the initial conditions for both Caputo fractional differential equations and ordinary differential equations take on the same form, but in real applications those of Caputo derivative are usually used since it has a clear physical meaning.

Definition 2.2 The two parameter Mittage-Leffler function $E_{\alpha, \beta}$ for $\alpha, \beta>0$ is a function defined by the following series

$$
E_{\alpha, \beta}(z)=\sum_{\rho=0}^{\infty} \frac{z^{\rho}}{\Gamma(\alpha \rho+\beta)}, z \in \square
$$

According to [34], for arbitrary n-th order square matrix A and $\alpha>0$, based on the definition of Mittage-Leffler function, the formula for the pseudo-transition matrix $\phi_{0}(t)$ of the linear fractionalorder system ${ }^{c} D^{\alpha} x(t)=A(t) x(t)$ is given as

$$
\phi_{0}(t)=E_{\alpha, \beta}\left(A t^{\alpha}\right)=\sum_{\rho=0}^{\infty} \frac{A^{\alpha} t^{\alpha \rho}}{\Gamma(\rho \alpha+1)}
$$

and then we set

$$
\phi(t)=t^{\alpha-1} E_{\alpha, \alpha}\left(A t^{\alpha}\right)=t^{\alpha-1} \sum_{\rho=0}^{\infty} \frac{A^{\alpha} t^{\alpha \rho}}{\Gamma((\rho+1) \alpha)}
$$

For $\alpha=1$, the pseudo-transition matrix is equivalent to the classical transition matrix of ordinary differential equations

$$
\phi_{0}(t)=\sum_{\rho=0}^{\infty} \frac{A^{\rho} t^{\rho}}{\Gamma(\rho+1)}=\sum_{\rho=0}^{\infty} \frac{(A t)^{\rho}}{\rho}=e^{A t}
$$

This implies that the pseudo-transition matrix $\phi_{0}(t)$ is also known as matrix $\alpha$-exponential function and is denoted by $\phi_{0}(t)=e_{\alpha}{ }^{A t}$ [21]. In the space of the $n$-th order matrix with real elements, it is convergent.

Definition 2.3: Let $f(t)$ be an n-dimensional vector-valued function, then the Laplace transform of a function is defined as

$$
F(s)=L[f(t)]=\int_{0}^{t} e^{-s t} f(t) d t, \quad s \in \square
$$

Note: if $0<\alpha<1$, then

$$
L\left[\left(D^{\alpha} f\right)(t)\right]=s^{\alpha} L[f(t)]-s^{\alpha-1} f(0)
$$

(see [11]).

Lemma 2.1: Let $\square$ be the complex plane, for any $\alpha>0, \beta>0$ and $A \in \square^{n \times n}$, then

$$
L\left[t^{\beta-1} E_{\alpha, \beta}\left(A t^{\alpha}\right)\right]=s^{\alpha-\beta}\left(s^{\alpha} I-A\right)^{-1}, \quad \square(s)>\|A\|^{\frac{1}{\alpha}}
$$

where $\square(s)$ represents the real part of the complex number $s, I$ denotes the identity matrix, and the inverse Laplace transform is given by following formula 


$$
\begin{gathered}
L^{-1}\left[s^{\alpha-1}\left(s^{\alpha} I-A\right)^{-1}\right]=\phi_{0}(t) \\
L^{-1}\left[\left(s^{\alpha} I-A\right)^{-1}\right]=\phi(t)
\end{gathered}
$$

(see [6])

Lemma 2.2 Let $0<\alpha<1$; If $f:[0, T] \rightarrow \square^{n}$ is continuous and exponentially bounded, then the solution of equ. (1) can be presented as

$$
x(t)=\phi_{0}(t) x(0)+\int_{0}^{t} \phi(t-s) \times(A x(s-\sigma(s))+B u(s)) d s, \quad t \in[0, T]
$$

and

Proof:

$$
x(t)=x(0), t \in\left[-\sigma_{m}, 0\right]
$$

Taking the Laplace transform of both sides of equ. (1) and using definition 2.2 and lemma 2.1, gives

$$
s^{\alpha} L[x(t)]-s^{\alpha-1} x(0)=A_{0} L[x(t)]+L\left[A_{1} x(t-\sigma(t))+B u(t)\right]
$$

Hence, equ. (5) can be written as

$$
\left(s^{\alpha} I-A_{0}\right) L[x(t)]=s^{\alpha-1} x(0)+L[A x(t-\sigma(t))+B u(t)]
$$

and

$$
\begin{aligned}
L[x(t)] & =\left(s^{\alpha} I-A_{0}\right)^{-1} s^{\alpha-1} x(0)+\left(s^{\alpha} I-A_{0}\right)^{-1} L[A x(t-\sigma(t))+B u(t)] \\
= & L\left[\phi_{0}(t) x(0)\right]+L[\phi(t)] L[A x(t-\sigma(t))+B u(t)]
\end{aligned}
$$

Now applying the convolution theorem of the Laplace transform we obtain

$$
L[x(t)]=L\left[\phi_{0}(t) x(0)\right]+L\left[\int_{0}^{t} \phi(t-s)(A x(s-\sigma(s)))+B u(s) d s\right]
$$

taking the inverse Laplace transform of both sides of equ. (7), we obtain

$$
x(t)=\phi_{0}(t) x(0)+\int_{0}^{t} \phi(t-s) \times(A x(s-\sigma(s))+B u(s)) d s
$$

as required.

\section{Main result}

For the fractional order equ. (1), we prove the necessary and sufficient conditions for the controllability criteria in this section.

\section{Definition 3.1}

The equ. (1) is said to be controllable on the time interval $[0, T]$ if for every $x_{0}, x_{1} \in \square^{n}$, there exist a control $u(t)$ such that the solution of the equ. (1) satisfies the condition $x(0)=x_{0}$ and $x(T)=x_{1}$

\section{Theorem 3.1}

The fractional order equ. (1) is relatively controllable on the time interval $[0, T]$ if and only if the $n \times n$ dimensional Grammian matrix

$$
W(0, T)=\int_{0}^{T} \phi(T-s) B B^{*} \phi^{*}(T-s) d s
$$

is nonsingular where $*$ denotes the matrix transpose and $\phi^{*}(t)=E_{\alpha, \alpha}\left(A * t^{\alpha}\right)$

\section{Proof:}

Suppose $W(0, T)$ is nonsingular, this means that there exists a well-defined inverse matrix $W^{-1}(0, T)$. For any initial complete state $x(0) \in C\left(\left[-\sigma_{M}, 0\right], \square^{n}\right)$ we take the following control function 


$$
\tilde{u}(t)=B^{*} \phi^{*}(T-t) W^{-1}(0, T)\left[\tilde{x}-\phi_{0}(t) x(0)-\int_{0}^{T} \phi(T-s) A x(s-\sigma(s)) d s\right]
$$

Substituting $t=b$ in equ. (8) we get

$$
x(b)=\phi_{0}(b) x(0)+\int_{0}^{b} \phi(b-s) A x(s-\sigma(s)) d s+\int_{0}^{b} \phi(b-s) B u(s) d s
$$

Now inserting equ. (10) in equ. (11) we obtain

$$
\begin{aligned}
& x(b)=\phi_{0}(b) x(0)+\int_{0}^{b} \phi(b-s) A x(s-\sigma(s)) d s \\
& +\int_{0}^{b} \phi(b-s) B B^{*} \phi^{*}(b-t) W^{-1}(0, b)\left[\tilde{x}-\phi_{0}(b) x(0)-\int_{0}^{b} \phi(b-s) A x(s-\sigma(s)) d s\right]=\tilde{x}
\end{aligned}
$$

By definition (3.1) the equ. (1) is relatively controllable in $[0, b]$.

We prove the necessary condition by contradiction. On the other hand, suppose that equ. (1) is relatively controllable, but the Grammian matrix $W(0, T)$ is singular. Then there exists a nonzero vector $y$ such that

$$
y^{*} W(0, T) y=0
$$

That is

$$
\int_{0}^{b} y^{*} \phi(b-s) B B^{*} \phi^{*}(b-s) d s=0
$$

Thus, for each $t \in[0, b]$, we have

$$
y^{*} \phi(b-t) B=0
$$

Since the system is controllable. By assumption, there exists an input $u$ such that it drives the initial state $x_{0}$ to the origin in the interval $[0, b]$. It follows that

$$
\begin{aligned}
x(b) & =x\left(b, x_{0}, u_{0}\right) \\
& =\phi_{0}(b) x(0)+\int_{0}^{b} \phi(b-s) A x(b-\sigma(s)) d s+\int_{0}^{b} \phi(b-s) B u_{0}(s) d s=0
\end{aligned}
$$

Moreover, there exists a control $\tilde{u}(t)$ that drives the initial state $x_{0}$ to the state $\tilde{x}$, hence

$$
\begin{aligned}
\tilde{x} & =x\left(b, x_{0}, \tilde{u}\right) \\
& =\phi_{0}(b) x(0)+\int_{0}^{b} \phi(b-s) A x(s-\sigma(s)) d s+\int_{0}^{b} \phi(b-s) B \tilde{u}(s) d s=0
\end{aligned}
$$

Combining equs. (15) and (16) gives

$$
\tilde{x}-\int_{0}^{b} \phi(b-s) B\left[\tilde{u}(s)-u_{0}(s)\right] d s=0
$$

Multiplying both sides of equ. (17) and using equ. (14) by $\tilde{x}^{\prime}$ gives

$$
\tilde{x} \tilde{x}^{\prime}-\tilde{x}^{\prime} \int_{0}^{b} \phi(b-s) B\left[\tilde{u}(s)-u_{0}(s)\right] d s=0
$$

By equ. (14), the second term of equ. (18) is zero, hence $\tilde{x} \tilde{x}^{\prime}=0$. Thus $\tilde{x}=0$, this contradicts the assumption. Therefore, the Grammian matrix $W(0, T)$ is nonsingular, hence the desired result.

The next theorem is a necessary and sufficient condition for the relative controllability of equ. (1) that is based on the matrices A and B. this is an algebraic criterion similar to the famous Kalman rank condition, see [30].

Theorem 3.2: the fractional control system equ. (1) is controllable in the interval $[0, T]$ if and only if $\operatorname{rank}\left[B, A B, A^{2} B, \ldots, A^{n-1} B\right]=n$

\section{Proof:}

According to Carley-Hamilton theorem $\phi(t)=t^{\alpha-1} E_{\alpha, \alpha}\left(A t^{\alpha}\right)$ can be represented as 


$$
\phi(t)=t^{\alpha-1} \sum_{\rho=0}^{\infty} \frac{A^{\rho} t^{\alpha \rho}}{\Gamma(\rho \alpha+\alpha)}=\sum_{\rho=0}^{\infty} \frac{t^{\rho \alpha+\alpha-1}}{\Gamma(\rho \alpha+\alpha)} A^{\rho}=\sum_{\rho=0}^{n-1} G_{\rho}(t) A^{\rho}
$$

For $b \in\left[0, t_{1}\right]$

$$
\begin{aligned}
x(b) & =\phi_{0}(b) x_{0}+\int_{0}^{b} \phi(b-s) \times[A x(s-\sigma(s))+B u(s)] d s \\
& =\phi_{0}(b) x_{0}+\sum_{\rho=0}^{n-1} \int_{0}^{b} G_{\rho}(b-s) A^{k} \times[A x(s-\sigma(s))+B u(s)] d s
\end{aligned}
$$

Let

$$
\psi=\phi_{0}(b) x_{0}+\sum_{\rho=0}^{n-1} \int_{0}^{b} G_{\rho}(b-s) A^{\rho} \times[A x(s-\sigma(s))] d s
$$

Then, subtracting equ. (21) from equ. (20) yields

$$
\begin{aligned}
x(b)-\psi & =\sum_{\rho=0}^{n-1} A^{\rho} B \int_{0}^{b} G_{\rho}(b-s) u(s) d s \\
& =\left[B, A B, A^{2} B, \ldots, A^{n-1} B\right]\left[\begin{array}{l}
c_{1} \\
c_{2} \\
c_{3} \\
\cdot \\
\cdot \\
\cdot \\
c_{n-1}
\end{array}\right]
\end{aligned}
$$

where $c_{\rho}=\int_{0}^{b} G_{\rho}(b-s) u(s) d s, \rho=0,1,2, \ldots, n-1$. Note that for arbitrary $x(0) \in C\left(\left[-\tau_{M}, 0\right], \square^{n}\right)$ and $x(b) \in \square^{n}$, the necessary and sufficient condition to have a control $u(t)$ satisfy equ. (22) is that

$$
\operatorname{rank}\left[B, A B, A^{2} B, \ldots, A^{n-1} B\right]=n
$$

For $b \in\left(t_{i}, t_{i+1}\right) \quad i=1.2, \ldots, k$

$$
\begin{aligned}
& x(b)=\phi_{0}(b) x_{0}+\sum_{j=1}^{i} I_{j}\left(x\left(\bar{t}_{j}\right)\right)+\int_{0}^{b} \phi(b-s) \times[A x(s-\sigma(s))+B u(s)] d s \\
& =\phi_{0}(b) x_{0}+\sum_{\rho=0}^{n-1} \int_{0}^{b} G_{\rho}(b-s) A^{\rho} \times[A x(s-\sigma(s))+B u(s)] d s
\end{aligned}
$$

Combining equ. (21) and equ. (23) yields

$$
\begin{aligned}
x(b)-\psi-\sum_{j=1}^{i} I_{j}\left(x\left(\bar{t}_{j}\right)\right) & =\sum_{\rho=0}^{n-1} A^{\rho} B \int_{0}^{b} G_{k}(b-s) u(s) d s \\
& =\left[B, A B, A^{2} B, \ldots, A^{n-1} B\right]\left[\begin{array}{l}
c_{0} \\
c_{1} \\
\cdot \\
\cdot \\
\cdot \\
c_{n-1}
\end{array}\right]
\end{aligned}
$$

Note that for arbitrary $x_{0} \in C\left(\left[-\sigma_{M}, 0\right], \square^{n}\right)$ and $x(b) \in \square^{n}$, the necessary and sufficient condition to have a control $u(t)$ satisfying equ. (24) is that 


$$
\operatorname{rank}\left[B, A B, A^{2} B, \ldots, A^{n-1} B\right]=n
$$

This completes the proof.

\section{Reference:}

[1] K. Balachandran, Controllability of Nonlinear Systems with Implicit Derivatives, IMA Journal of Mathematical Control and Information, 5(2)(1988), 77-83.

[2] K. Balachandran \& J. Kokila, On the controllability of fractional dynamical systems. International Journal of Applied Mathematics and Computer Science, 22(3)(2012), 523-531. https://doi.org/10.2478/v10006-012-0039-0

[3] K. Balachandran \& J. P. Dauer, Controllability of Nonlinear Systems via Fixed point Theorem, Journal of Optimization Theory and Applications, 5(3)(1987), 345- 352.

[4] K. Balachandran \& P. Balasubramaniam, A Note on Controllability of Nonlinear Volterra Integrodifferential Systems, Kybernetika, 28(4)(1992), 284-291.

[5] K. Balachandran \& P. Balasubramaniam, Controllability of Nonlinear Neutral Volterra Integrodifferential Systems, Journal of Australian Mathematical Society, 36(1)(1994), 107-116, https://doi.org/10.1017/s0334270000010274

[6] K. Balachandran\& S. Divya, Controllability of nonlinear implicit fractional integrodifferential systems, International Journal of Applied Mathematics and Computer Science, 24(4)(2014), 713-722. https://doi.org/10.2478/amcs-2014-0052

[7] K. Balachandran, V. Govindaraj, M. Rivero \& J. J. Trujillo, Controllability of fractional damped dynamical systems, Applied Mathematics and Computation, 257(2015), 66-73. https://doi.org/10.1016/j.amc.2014.12.059

[8] K. Balachandran, Y. Zhou \& J. Kokila, Relative controllability of fractional dynamical systems with distributed delays in control, Computers \& Mathematics with Applications, 64(10)(2012), 3201-3209. https://doi.org/10.1016/j.camwa.2011.11.061

[9] A. Bensoussan, G. daPrato, M. C. Delfour \& S. K. Mitter, Representation and Control of Infinite Dimensional System, Vol. 1 and II, Birkhauser, Boston,(1993)

[10] M. Caputo, Linear models of dissipation whose Q is almost frequency independent, II. Geophys. J. R. Astron. Soc. 13(5)(1967), 529-539, https://doi.org/10.1111/j.1365-246x.1967.tb02303.x

[11] A. Dabbouchi \& D. Baleanu, Exact Null Controllability for Fractional Nonlinear Integrodifferential equations via Implicit Evolution systems, Journal of Applied Mathematics, Vol(2012), Article ID 931975, 1-17, https://doi.org/10.1155/2012/931975

[12] C. Dacka, On the Controllabilityof a Class of Nonlinear Systems, IEEE Transactions on Automatic Control, 25(2)(1980), 263-266.

[13] S. Das, Functional fractional calculus, academic press, San Diego, (1999).

[14] I. Davies \& P. C. Jackreece, Controllability and Null Controllability of Linear Systems, J. Appl. Sc. Environ. Mgt, 9(3)(2005), 31-38, https://doi.org/10.4314/jasem.v9i3.17349

[15] N. M. F. Ferreira, F. B. Duarte, F. M. Miguel, M. G. Marcos \& J. A. T. Machado, Application of fractional calculus in the Dynamical analysis and control of mechanical manupulators, Fractional calculus and applied analysis,11(1),2008, 91-113. 
[16] V. Govindaraj \& R. K. George, Controllability of fractional dynamical systems: A functional analytic approach, Mathematical Control and Related Fields, 7(4)(2017), 537-562. https://doi.org/10.3934/mcrf.2017020

[17] T. L. Guo, Controllability and Observability of Impulsive Fractional Linear Time- Invariant Systems, Computers and Mathematics with Applications, 64(10)(2012), 3171-3182, https://doi.org/10.1016/j.camwa.2012.02.020

[18] R. Hilfer, Application of fractional calculus in Physics, World Scientific, Singapore,(2000).

[19] P. C. Jackreece, Controllability of functional differential equation with infinite delay, International Journal of Mathematics and Statistics Invention (IJMSI), 2(4)(2014), 58-66.

[20] R. E. Kalman, Y. C. Ho, \& K. S. Narendra, Controllability of Linear Dynamical Systems, Contributions to Differential Equations, 1(2)(1963), 189-213.

[21] A. A. Kilbas, H. M. Srivastava \& J. J. Trujillo, Theory and applications of fractional differential equations, Elsevier, New York,(2006).

[22] J. Klamka, Constrained Controllability of Dynamical systems, International Journal of Applied Mathematics and Computer science, 9(2)(1999), 231-244.

[23] J. Klamka, Controllability of Dynamical Systems, Mathematics and Computers in Simulation, 34(1)(1992), 85, https://doi.org/10.1016/0378-4754(92)90107-R

[24] J. Klamka, Controllability of Dynamical Systems- A survey, Arch. Control Sci., 2(2/4)(1993), 281-307.

[25] J. Klamka, Controllability of Dynamical Systems. A survey. Bulletin of the Polish Academy of Sciences, 61(2)(2013), 335-342, https://doi.org/10.2478/bpasts-2013-0031

[26] J. Klamka, Controllability of Retarded Dynamical Systems, Kybernetika, 32(6)(1996), 591-600.

[27] J. Klamka, On the Global Controllability of Perturbed nonlinear Systems, IEEE Transactions on Automatic Control, 20(1),(1975), 170-172, https://doi.org/10.1109/tac.1975.1100870

[28] J. Klamka, On the Local Controllability of Perturbed Nonlinear Control systems, IEEE Transactions on Automatic control, 20(2)(1975), 289-291, https://doi.org/10.1109/tac.1975.1100929

[29] J. Klamka, Constrained Controllability of Semilinear Systems with Delayed Controls, Bulletin of the Polish Academy of Science: Technical Science, 56(4)(2008), 333-337.

[30] J. Klamka, Controllability of Dynamical System, Kluwer Academic Publisher, Dordrecht,(1991)

[31] V. V. Kulish \& J. L. Lage, Application of fractional calculus to Mechanics, Journal of Fluids Engineering, 124(3)(2002), 803-806.

[32] N. I. Mahmudov, Approximate Controllability of Fractional Sobolev-type Evolution Equation in Banach Spaces, Abstract and Applied analysis, (2013),1-9, https://doi.org/10.1155/2013/502839

[33] F. A. Mohd, S. Manoj \& J. Renu, An application of fractional calculus in electrical engineering, Advanced Engineering Technology and Application, 5(2), 2016, 41-45, https://doi.org/10.18576/aeta/050204

[34] C. Monje, Y. Q. Chen, B. M. Vinagre, D. Xue, \& V. Feliu, Fractional-order Systems and Control: Fundamentals and Applications, Springer, London,(2010).

[35] M. Naber, Time fractional Schrodinger equation, J. Maths. Phys. 45(2004), 3339- 3352.

[36] K. B. Oldham \& J. Spanier, The fractional calculus, Academic Press, New York,(1974). 
[37] I. Podlubny, Fractional differential equation, Academic Press, San Diego, (1999).

[38] Y. Ryabov \& A. Puzenko, Damped oscillation in view of the fractional oscillator equation, Phys. Rev., 66(2002), 184-201, https://doi.org/10.1103/physrevb.66.184201

[39] J. Sabatier, O. P. Agarwal \& J. A. Tenreiro Machado, Advances in Fractional Calculus: Theoretical Developments and Applications in Physics and Engineering, Springer-Verlag, New York, N.Y,(2007).

[40] S. G. Samko, A. A. Kilbas \& O. I. Marichev, Fractional integrals and derivatives theory and applications, Gordon and Breach, New York,(1993).

[41] B. Sikora, Controllability criteria for time-delay fractional systems with a retarded state, International Journal of Applied Mathematics and Computer Science, 26(3), 2016, 521-531. https://doi.org/10.1515/amcs-2016-0036

[42] E. Soczkiewicz, Application of fractional calculus in the theory of viscoelasticity, Molecular and Quantum Acoustics, 23(2002), 397-404.

[43] W. M. Wonham \& A. Morse, Decoupling and Pole assignment in Linear Multivariable Systems: A Geometric Appraoch, SIAM Journal of Control and Optimization, 8(1)(1970), 1-18, https://doi.org/10.1137/0308001

[44] D. J. N. Xiao-Li, Controllability of Nonlinear Fractional Delay Dynamical Systems with prescribed controls, Nonlinear Analysis: Modelling and Control, 23(1)(2018), 1-18, https://doi.org/10.1016/S00344877(16)30007-6

[45] F. Yu, Integrable coupling system of fractional differential equations, Eur. Phys. J. Spec. Top., 193, 2011, 27-47.

[46] W. K. Zahra, M. M. Hikal \& A. B. Taher, Solution for fractional order electrical circuits in Laplace transform and on standard finite difference method, Journal of the Egyptian Mathematical Society, 25(2) (2017), 252-261, https://doi.org/10.1016/j.joems.2017.01.007

[47] H. Zhang, J. Cao \& W. Jiang, Controllability criteria for Linear Fractional Differential Systems with State Delay and Impulse, Journal of Applied Mathematics,(2013), https://doi.org/10.1155/2013/146010 